\title{
Can the silkworm (Bombyx mori) be used as a human disease model?
}

\author{
Hiroko Tabunoki ${ }^{1, *}$, Hidemasa Bono ${ }^{2}$, Katsuhiko Ito ${ }^{1}$, Takeshi Yokoyama ${ }^{1}$ \\ ${ }^{1}$ Department of Biological Production, Faculty of Agriculture, Tokyo university of Agriculture and Technology, Tokyo, Japan; \\ ${ }^{2}$ Database Center for Life Science (DBCLS), Research Organization of Information and Systems (ROIS), Mishima, Shizuoka, Japan.
}

\begin{abstract}
Summary Bombyx mori (silkworm) is the most famous lepidopteran in Japan. B. mori has long been used in the silk industry and also as a model insect for agricultural research. In recent years, $B$. mori has attracted interest in its potential for use in pathological analysis of model animals. For example, the human macular carotenoid transporter was discovered using information of $B$. mori carotenoid transporter derived from yellow-cocoon strain. The $B$. mori carotenoid transport system is useful in human studies. To develop a human disease model, we characterized the human homologs of $B$. mori, and by constructing KAIKO functional annotation pipeline, and to analyze gene expression profile of a unique B. mori mutant strain using microarray analysis. As a result, we identified a novel molecular network involved in Parkinson's disease. Here we describe the potential use of a spontaneous mutant silkworm strain as a human disease model. We also summarize recent progress in the application of genomic information for annotation of human homologs in $B$. mori. The $B$. mori mutant will provide a clue to pathological mechanisms, and the findings will be helpful for the development of therapies and for medical drug discovery.
\end{abstract}

Keywords: Silkworm, human disease model, Bombyx mori mutant, Parkinson's disease, translucent larval skin

\section{Introduction}

The silkworm (Bombyx mori), which produces silk fiber in its silk glands, is the most famous lepidopteran in Japan. It was domesticated by humans more than 5,000 years ago in China. B. mori cannot survive without human help.

In the 19th century, silkworm was an important livestock animal in Japan. Nature and Science Museum of Tokyo University of Agriculture and Technology (http://www.tuat.ac.jp/ museum/) displays several ukiyo-e that depicts scenes of sericulture at that time. One ukiyo-e depicts women reeling cocoons (Figure $1 \mathrm{~A})$, and another depicts women rearing silkworms (Figure 1B). Thus, rearing silkworms, reeling cocoons

Released online in J-STAGE as advance publication February 7, 2016.

*Address correspondence to:

Dr. Hiroko Tabunoki, Department of Biological Production, Faculty of Agriculture, Tokyo university of Agriculture and Technology, 3-5-8 Saiwai-cho, Fuchu, Tokyo 183-8509, Japan.

E-mail: h_tabuno@cc.tuat.ac.jp and weaving cloth were done by the women. These methods of sericulture were recorded in some books. Silkworms were important possessions for the Japanese people. They preferred colored to white cocoons, and variety of colored cocoons were produced by Japanese sericultureists till the 19th century. However, it has not been clear why whited cocoon strains were standardized at the early 20th century. The Japanese people collected many silkworm strains from the Europe and China. Because Japanese sericultureists were seeking for silkworm strains which were disease-resistant and made the big cocoon. In this process, Japanese sericultureists obtained various phenotypes, and discovered many unique mutants. Therefore, Japanese sericultureists have maintained these many silkworm races of these for the future gene resource need.

Additionally, Japanese geneticist's Toyama rediscovered the Mendel's law of heredity in animal using white and yellow cocoon race of B. mori in 1906 (1). Thereby, the phenotypes of $B$. mori were genetically linked to the causative genes on the linkage maps of $B$. mori. Currently, 456 mutant strains are maintained at the National Bio Resource Project (NBRP) KAIKO (http:// 


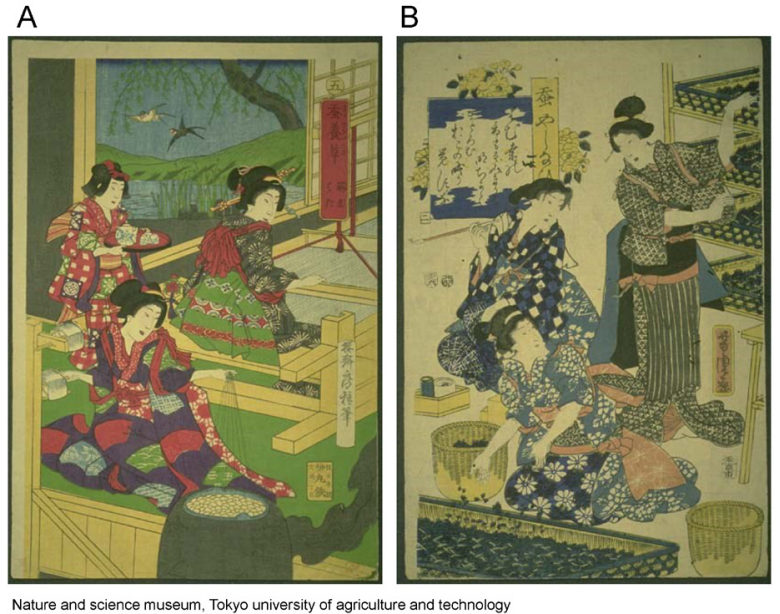

Figure 1. Sericulture and silkworm depiction on Ukiyoe.

silkworm.nbrp.jp/) located at Kyushu University.

The silkworm has been used as a model insect for agricultural research for several reasons: $i$ ) the majority of agricultural pests are lepidopterans, $i i$ ) its genome sequence is well characterized, iii) various genetic mutants are available, and $i v$ ) silkworm is amenable to transgenic, knock-out, and microarray technologies (27). However, to date, silkworms have never been used as human disease models.

Here we describe the potential use of a spontaneous silkworm mutant strain as a human disease model, based on our current study (8). We also summarize recent progress in the application of genomic information for the annotation of human homologs in B. mori. These findings will help elucidate molecular mechanisms of human diseases.

\section{The human macular carotenoid transporter discovered using an information of $B$. mori}

There are several known colored cocoons: yellow, pink, golden yellow, straw, green, and sasa (yellowish green). Yellow-, pink-, golden-yellow-, and flesh-colored cocoon pigments are derivatives of carotenoids (9), whereas sasaand green-cocoon pigments are derivatives of flavonoids $(10,11)$. These pigments protect pupae from sunlight as antioxidants (12). A carotenoid-binding protein (CBP) was identified in a B. mori yellow-cocoon strain in 2002 (13) as an intracellular carotenoid transporter in the silk gland by a biochemical approach. Interestingly, CBP was categorized as the first member of a steroidogenic acute regulatory protein (StAR) family whose members bind only carotenoids in their lipid-binding domains.

Landrum et al. (14) have reported that carotenoids are accumulated in the human macula as macular pigment. A low concentration of macular pigment increases the risk of age-related macular degeneration from blue light damage. However, sustained intake of dietary carotenoids may reduce the risk of age-related human macular degeneration (14). Thus, identification of a macular carotenoid transporter is important for elucidation of the molecular mechanism of macular degeneration.

The antibody to $B$. mori CBP has cross-reactivity to macular carotenoid transporter identified from human retina and layers of the primate macula where the macular carotenoid pigment is at its highest concentration (15). The human macular carotenoid transporter StARD3 (also known as MLN64) was discovered by Bernstein et al. in 2009 (16).

The B. mori CBP information and antibody were helpful for identification of the human macular carotenoid transporter. Therefore, the common molecular mechanisms were present in the carotenoid transport system between $B$. mori and human.

In the present study, a B. mori cocoon-color mutant was helpful for elucidation of the molecular mechanism of age-related human macular degeneration (17).

\section{How many human orthologs does silkworm have?}

A draft silkworm genome sequence was completed by Chinese and Japanese groups in 2004 (2,3). In 2013, Suetsugu et al. (4) reported that the silkworm genome contained 16,823 gene loci, based on sequence analysis of cDNA data set. However, these data are not included in public databases currently. We accordingly identified human homologs using the Ensembl Metazoa (18) predicted-protein data set of $B$. mori.

There are challenges in using silkworm genome information. Silkworm genome sequences have been analyzed, but the functional annotation of the genes still remains obscure. If we can annotate the $B$. mori genome in the same manner as the Drosophila melanogaster genome, we can use the public databases to mammalian model organisms and commercial pathway softwares for microarray analysis or next-generation sequencing (NGS) data. Finally, we can analyze these big data from microarray or NGS analysis as deeply as for mammalian model organisms. D. melanogaster has been used as a human disease model in studies involving genegene interactions. A systematic BLAST search (19) revealed 548 human disease-associated genes in $D$. melanogaster. Importantly, D. melanogaster is the only insect species in which gene annotations have been extensively assigned $(20,21)$, and many human disease models have been developed. However, the analysis of human disease-associated genes was reported by Reiter et al. (19) in 2001 and the complete human genome was not available at that time. Thus, we needed the re-analysis of updated human gene set in the current study. To annotate silkworm genome information, we identified human homologs common to B. mori and D. melanogaster; we obtained the cDNA sequence sets of these species from Ensembl Metazoa, and we performed systematic BLAST search to identify human homologs in these species. B. mori contained 8,469 
Table 1. Comparison of human homologs between Bombyx mori and Drosophila melanogaster

\begin{tabular}{|c|c|c|c|c|c|}
\hline Species & Human homolog transcripts & Total transcripts & Human homolog genes & Total (genes) & Ratio (\%) of human homolog/total genes \\
\hline B. mori & 8469 & 14623 & 8469 & 14623 & 58 \\
\hline D. melanogaster & 21230 & 30362 & 8815 & 13918 & 63 \\
\hline
\end{tabular}

Table 2. Gene enrichment analysis of human homolog genes in Bombyx mori

\begin{tabular}{llll}
\hline KEGG ID & KEGG pathway & Count & $p$ value \\
\hline hsa05016 & Huntington's disease & 82 & $2.1 \mathrm{E}-4$ \\
hsa05010 & Alzheimer's disease & 68 & $1.1 \mathrm{E}-2$ \\
hsa05012 & Parkinson's disease & 51 & $1.1 \mathrm{E}-2$ \\
hsa04120 & Ubiquitin mediated proteolysis & 67 & $7.0 \mathrm{E}-5$ \\
hsa00190 & Oxidative phosphorylation & 54 & $2.5 \mathrm{E}-2$ \\
hsa03050 & Proteasome & 31 & $8.7 \mathrm{E}-6$ \\
\hline
\end{tabular}

4,020 genes shared with 57 Kyoto Encyclopedia of Genes and Genomes pathways.

and $D$. melanogaster contained 8,815 human homologs (Table 1). Thus, B. mori had $58 \%$ of the human homologs in the genes. Furthermore, we characterized these human homologs in $B$. mori by enrichment analysis using the DAVID bioinformatics database (https://david.ncifcrf.gov/home.jsp). The human homologs were included in 57 Kyoto Encyclopedia of Genes and Genomes (KEGG) pathways, and the genes in the most conserved pathways in the DAVID analysis were concerned in neurodegenerative disease, oxidative stress, and protein degradation-associated genes (Table 2). In addition, these pathways were also conserved in D. melanogaster, and corresponding related human disease models were developed. The FlyBase Human Disease Model Report List (http://flybase.org/static pages/FBhh/browse.html) contains 59 human disease models in $D$. melanogaster. The basic information of human disease related genes were obtained from Online Mendelian Inheritance in Man database. Human disease related fly ortholog was modified as functional disturbance, and the genetically modified fly was created. These human disease model flies are reported in the public database and stock centers. Accordingly, $B$. mori may have potential use as a human disease model similar to D. melanogaster.

\section{Abundance of spontaneous silkworm mutant stocks in Japan}

The NBRP KAIKO also maintains 456 spontaneous mutant $B$. mori strains. These genes responsible for each mutation have been mapped in the $B$. mori linkage map information. User can choose the mutant which correspond to gene symbols and mutant strains on the NBRP web site (http://www.shigen.nig.ac.jp/ silkwormbase/ViewCausativeGene.do?). Some causative genes of the product have been discovered and are shown in a table on the NBRP web site. However, many causative genes in $B$. mori mutants have not been identified. Importantly, spontaneous mutants have unique phenotypes that do not appear in other model species. For instance, there are a variety of cocoon colors, egg colors, and larval skin colors and patterns; translucent larval skin; and black-colored adults. If we can identify the causal genes responsible for these phenotypes as human homolog, the discovery will contribute to identify novel molecular mechanisms that could not be detected using other model organisms.

Thus, $B$. mori has many valuable spontaneous mutants in Japan. We propose that B. mori is a good human disease model candidate, on the basis of these spontaneous mutants.

\section{KAIKO functional annotation pipeline is useful for screening target molecules}

B. mori has 26 mutants in uric acid metabolism. The common phenotype of these mutants is translucent larval skin (22). Eight genes have been identified as causative genes in translucent larval skin mutants (2331 ). These genes are involved in the synthesis or uptake of uric acid.

To analyze the $B$. mori translucent larval skin mutant strain o751 (op), we constructed KAIKO functional annotation pipeline using corresponding information of B. mori human homologs and human genes (Figure 2).

The $B$. mori op mutant is classed as a translucent larval skin mutant (Japanese name, aburako) and displays occasional unique actions such as vibration. Classical linkage analysis has shown that the $o p$ gene is located on chromosome 23, and involved in the phenotypes of the extraordinarily high mortality, particularly in the pupal stage, and the male infertility except for the oily mutation (NBRP silkworm database; http://www.shigen.nig. ac.jp/silkwormbase/ViewStrainDetail.do?id=309). We investigated gene expression in the $B$. mori op mutant using microarray analysis with KAIKO functional annotation pipeline. We identified a novel uric acid synthesis-modulated pathway (Figure 2, gray-colored molecule) (8).

\section{Parkinson's disease and uric acid}

Parkinson's disease (PD) remains an incurable disease. Its mechanisms responsible for dopaminergic neuronal cell degradation cause oxidative stress or protein accumulation by ubiquitin proteasome failure, and this damage depletes dopamine levels in substantia 


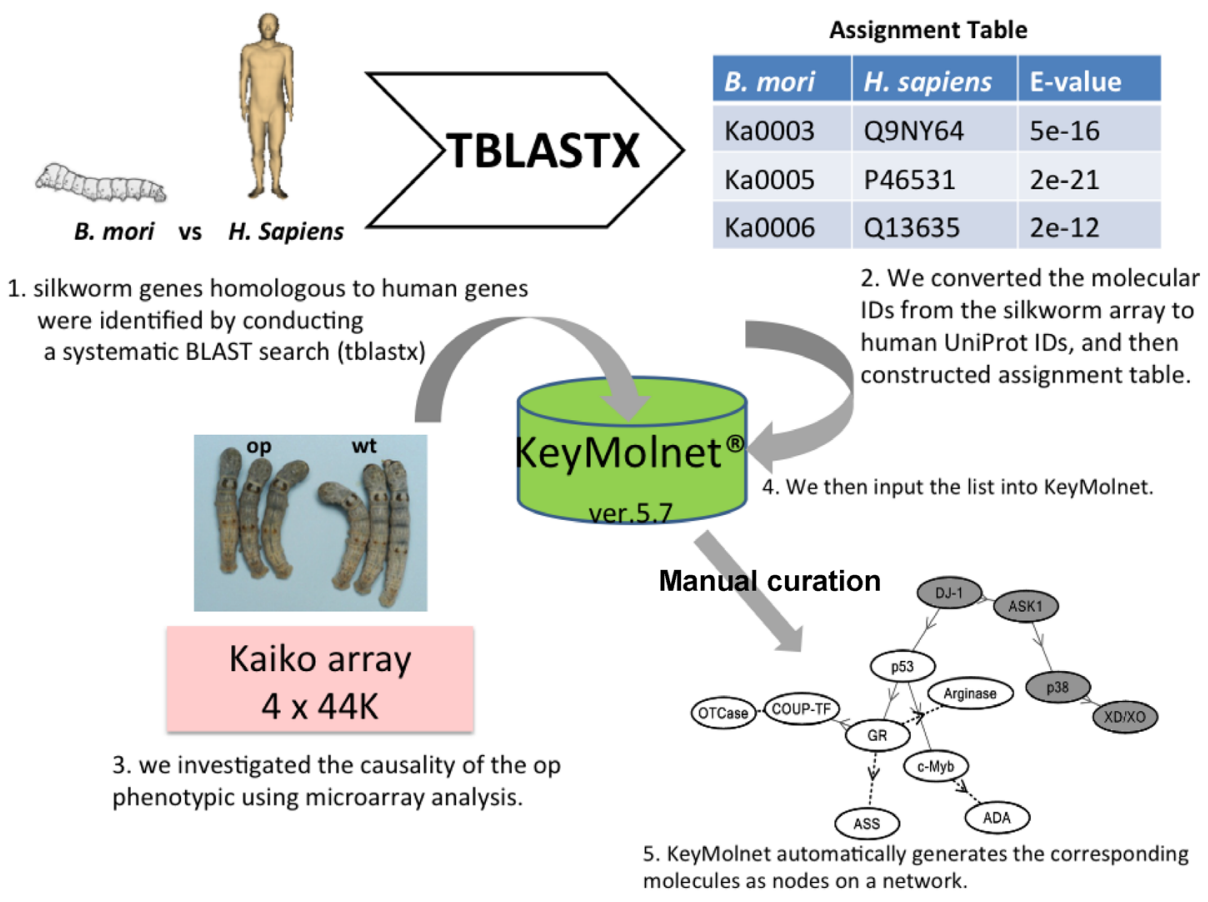

Figure 2. Screening of target molecule using KAIKO functional annotation pipeline.

nigra neurons $(32,33)$. Genetic studies have identified 21 genes associated with PD at different loci based on family linkage analysis [PD; Online Mendelian Inheritance in Man 168600]. PD-associated gene knockout animal models have been developed as familial PD models (34).

The majority of sporadic PD onset is caused by environmental stress $(35,36)$, and a molecular mechanism of oxidative stress has been developed. In animal models of sporadic PD, oxidative stress has been simulated using mitochondrial complex I inhibitors (37).

The final product of purine metabolism, uric acid, plays an important role as a physiological antioxidant (38). Several groups have reported a correlation between decreased plasma uric acid concentrations and clinical progression and stage of PD (39-45). Conversely, high plasma uric acid concentrations in hyperuricemia may reduce the risk and delay the progression of $\mathrm{PD}$, but it increases the risk of cardiac diseases (46). Thus, uric acid has a dual function in organisms. In the case of PD, uric acid may be expended to resist oxidative injury (47); however, the molecular mechanism underlying the decrease in plasma uric acid concentration in advanced clinical stages of PD has not been analyzed using either of these model animals. Owing to the lack of adequate animal models, the function of uric acid in PD pathogenesis is poorly understood.

\section{Why is a silkworm model a good candidate for the analysis of Parkinson's disease and uric acid?}

It is well established that the uric acid metabolism process uses $B$. mori mutants. Uric acid is synthesized mainly in the fat body and is thereafter transported to the integument via the hemolymph. It is the end product of purine degradation via xanthine/hypoxanthine reactions catalyzed by xanthine dehydrogenase. It is eliminated through the Malpighian tubules. Uric acid accumulates as urate granules and produces a whitening of the integument. In translucent larval skin mutants, it shows abnormal accumulation in the integument (22).

Uric acid plays a protective role against photooxidative stress in $B$. mori, as shown by a markedly reduced survival rate in larvae under UV irradiation with injection of allopurinol, an inhibitor of uric acid synthesis (48). It directly scavenges oxygen radicals and may play an important role in protection against environmental oxidative stress in B. mori.

Only B. mori translucent larval skin mutants show abnormality in integument color in the larval developmental stage. Other model organisms do not show a similar phenotype.

In human studies, plasma uric acid concentrations decrease following the clinical progression and stage development of PD (39-45). However, molecular mechanisms underlying reduction in plasma uric acid concentrations remain unknown. Reduced plasma uric acid concentrations are due to consumption of uric acid as an antioxidant in PD. Furthermore, the causative gene of human PD induces strong oxidative stress in the central nervous system (47). Moreover, the regulation of xanthine dehydrogenase phosphorylation in the uric acid synthesis pathway is unclear.

We accordingly investigated gene expression in the B. mori op mutant using KAIKO functional annotation pipeline for analysis of microarray data. We identified 
a novel uric acid synthesis-modulating pathway (Figure 2 ). We speculated that these molecules relate to the phosphorylation of the protein $(8)$. However, we were unable to identify the $o p$ causative gene in the present study.

Molecular mechanisms associating decreased plasma uric acid concentrations with PD remain obscure. $B$. mori translucent larval skin mutants provide promising clues for elucidation of these mechanisms and for development of therapies and drugs for PD. Further study of genes with common function in the uric acid synthesis pathways of humans and B. mori is warranted.

Do you now believe that silkworms can be used as human disease models?

\section{Acknowledgements}

We thank Dr. Takahiro Iino from Nature and science Museum, Tokyo University of Agriculture and Technology for advising, history, and ukiyo-e in the silkworm. This work was supported by Funds by the Japan Society for the Promotion of Science (JSPS) Grant-in-Aid for Scientific Research C (26450465).

\section{References}

1. Toyama K. On some silkworm crosses, with special reference to Mendel's law of heredity. Bull Coll Agr Tokyo Univ. 1906; 7:259-393.

2. Mita K, Kasahara M, Sasaki S, et al. The genome sequence of silk- worm, Bombyx mori. DNA Res. 2004; 11:27-35.

3. Xia Q, Zhou Z, Lu C, et al. A draft sequence for the genome of the domesticated silkworm (Bombyx mori). Science. 2004; 306:1937-1940.

4. Suetsugu Y, Futahashi R, Kanamori H, et al. Large scale full-length cDNA sequencing reveals a unique genomic landscape in a lepidopteran model insect, Bombyx mori. G3 (Bethesda). 2013; 9:1481-1492.

5. Tomita M, Munetsuna H, Sato T, Adachi T, Hino R, Hayashi M, Shimizu K, Nakamura N, Tamura T, and Yoshizato K. Transgenic silkworms produce recombinant human type III procollagen in cocoons. Nat Biotechnol. 2003; 21:52-56.

6. Wang F, Ma S, Xu H, Duan J, Wang Y, Ding H, Liu Y, Wang X, Zhao P, Xia Q. High-efficiency system for construction and evaluation of customized TALENs for silkworm genome editing. Mol Genet Genomics. 2013; 288:683-690.

7. Xia Q, Cheng D, Duan J, Wang G, Cheng T, Zha X, Liu C, Zhao P, Dai F, Zhang Z, He N, Zhang L, Xiang Z. Microarray-based gene expression profiles in multiple tissues of the domesticated silkworm, Bombyx mori. Genome Biol. 2007; 8:R162.

8. Tabunoki H, Ono H, Ode H, Ishikawa K, Kawana N, Banno Y, Shimada T, Nakamura Y, Yamamoto K, Satoh J, and Bono H. Identification of key uric acid synthesis pathway in a unique mutant silkworm Bombyx mori model of Parkinson's disease. PLOS One. 2013; 8:e69130.

9. Harizuka M. Physiological genetics of the carotenoids in
Bombyx mori with special reference to the pink cocoon. Bull Sericul Exp Stat. 1953; 14:141-156.

10. Tamura Y, Nakajima K, Nagayasu K, and Takabayashi C. Flavonoid 5-glucosides from the cocoon shell of the silkworm, Bombyx mori. Phytochemistry. 2002; 59:275278.

11. Kurioka A, Yamazaki M. Purification and identification of flavonoids from the yellow green cocoon shell (Sasamayu) of the silkworm, Bombyx mori. Biosci Biotechnol Biochem. 2002; 66:1396-1399.

12. Daimon T, Hirayama C, Kanai M, Ruike Y, Meng Y, Kosegawa E, Nakamura M, Tsujimoto G, Katsuma $\mathrm{S}$, Shimada T. The silkworm Green b locus encodes a quercetin 5-O-glucosyltransferase that produces green cocoons with UV-shielding properties. Proc Natl Acad Sci USA. 2010; 107:11471-11476.

13. Tabunoki H, Sugiyama H, Tanaka Y, Fujii H, Banno Y, Jouni ZE, Kobayashi M, Sato R, Maekawa H, Tsuchida $\mathrm{K}$. Isolation, characterization, and cDNA sequence of a carotenoid binding protein from the silk gland of Bombyx mori larvae. J Biol Chem. 2002; 277:32133-32140.

14. Landrum JT, Bone RA, Joa H, Kilburn MD, Moore LL, Sprague KE. A one year study of the macular pigment: The effect of 140 days of a lutein supplement. Exp Eye Res. 1997; 65:57-62.

15. Bhosale P, Li B, Sharifzadeh M, Gellermann W, Frederick JM, Tsuchida K, Bernstein PS. Purification and partial characterization of a lutein-binding protein from human retina. Biochemistry. 2009; 48:4798-4807.

16. Li B, Vachali P, Frederick JM, Bernstein PS. Identification of StARD3 as a lutein-binding protein in the macula of the primate retina. Biochemistry. 2011; 50:2541-2549.

17. Tsuchida K, Sakudoh T. Recent progress in molecular genetic studies on the carotenoid transport system using cocoon-color mutants of the silkworm. Arch Biochem Biophys. 2015; 15:151-157.

18. Kersey PJ, Allen JE, Armean I, et al. Ensembl Genomes 2016: More genomes, more complexity. Nucleic Acids Res. 2016; 44(D1):D574-580.

19. Reiter LT, Potocki L, Chien S, Gribskov M, Bier E. A systematic analysis of human disease-associated gene sequences in Drosophila melanogaster. Genome Res. 2001; 11:1114-1125.

20. dos Santos G, Schroeder AJ, Goodman JL, Strelets VB, Crosby MA, Thurmond J, Emmert DB, Gelbart WM. FlyBase: Introduction of the Drosophila melanogaster Release 6 reference genome assembly and large-scale migration of genome annotations. Nucleic Acids Res. 2015; 43(Database issue):D690-697.

21. Matthews BB, Dos Santos G, Crosby MA, Emmert DB, St Pierre SE, Gramates LS, Zhou P, Schroeder AJ, Falls K, Strelets V, Russo SM, Gelbart WM, FlyBase Consortium. Gene Model Annotations for Drosophila melanogaster: Impact of High-Throughput Data. G3 (Bethesda). 2015; 5:1721-1736.

22. Kômoto N. Silkworm mutants whose deficiency of epidermal urate granules causes translucent larval skin phenotypes. Tanpakushitsu Kakusan Koso. 2004; 49:21982205. (in Japanese)

23. Fujii T, Banno Y, Abe H, Katsuma S, Shimada T. A homolog of the human Hermansky-Pudluck syndrome-5 (HPS5) gene is responsible for the oa larval translucent mutants in the silkworm, Bombyx mori. Genetica. 2012; 140:463-468.

24. Fujii T, Abe H, Katsuma S, Mita K, Shimada T. Mapping 
of sex-linked genes onto the genome sequence using various aberrations of the $\mathrm{Z}$ chromosome in Bombyx mori. Insect Biochem Mol Biol. 2008; 38:1072-1079.

25. Fujii T, Daimon T, Uchino K, Banno Y, Katsuma S, Sezutsu H, Tamura T, Shimada T. Transgenic analysis of the BmBLOS2 gene that governs the translucency of the larval integument of the silkworm, Bombyx mori. Insect Mol Biol. 2010; 19:659-667.

26. Wang L, Kiuchi T, Fujii T, Daimon T, Li M, Banno Y, Kikuta S, Kikawada T, Katsuma S, Shimada T. Mutation of a novel $\mathrm{ABC}$ transporter gene is responsible for the failure to incorporate uric acid in the epidermis of ok mutants of the silkworm, Bombyx mori. Insect Biochem Mol Biol. 2013; 43:562-571.

27. Kômoto N, Sezutsu H, Yukuhiro K, Banno Y, Fujii $\mathrm{H}$. Mutations of the silkworm molybdenum cofactor sulfurase gene, og, cause translucent larval skin. Insect Biochem Mol Biol. 2003; 33:417-427.

28. Kômoto N. A deleted portion of one of the two xanthine dehydrogenase genes causes translucent larval skin in the oq mutant of the silkworm (Bombyx mori). Insect Biochem Mol Biol. 2002; 32:591-597.

29. Kiuchi T, Banno Y, Katsuma S, Shimada T. Mutations in an amino acid transporter gene are responsible for sexlinked translucent larval skin of the silkworm, Bombyx mori. Insect Biochem Mol Biol. 2011; 41:680-687.

30. Wang L, Kiuchi T, Fujii T, Daimon T, Li M, Banno Y, Katsuma S, Shimada T. Reduced expression of the dysbindin-like gene in the Bombyx mori ov mutant exhibiting mottled translucency of the larval skin. Genome. 2013; 56:101-108.

31. Ito K, Katsuma S, Yamamoto K, Kadono-Okuda K, Mita $\mathrm{K}$, Shimada T. A 25bp-long insertional mutation in the BmVarp gene causes the waxy translucent skin of the silkworm, Bombyx mori. Insect Biochem Mol Biol. 2009; 39:287-293.

32. Devine MJ, Plun-Favreau H, Wood NW. Parkinson's disease and cancer: Two wars, one front. Nat Rev Cancer. 2011; 11:812-823.

33. Dias V, Junn E, Mouradian MM. The role of oxidative stress in Parkinson's disease. J Parkinsons Dis. 2013; 3:461-491.

34. Hatano T, Kubo S, Sato S, Hattori N. Pathogenesis of familial Parkinson's disease: New insights based on monogenic forms of Parkinson's disease. J. Neurochem. 2009; 111:1075-1093.

35. Warner TT, Schapira AH. Genetic and environmental factors in the cause of Parkinson's disease. Ann Neurol. 2003; 53 (Suppl 3):S16-23; discussion S23-5.
36. Montgomery EB Jr, Baker KB, Lyons K, Koller WC. Abnormal performance on the PD test battery by asymptomatic first-degree relatives. Neurology. 1999; 52:757-762.

37. Bove J, Prou D, Perier C, Przedborski S. Toxin-induced models of Parkinson's disease. NeuroRx. 2005; 2:484-494.

38. Ames BN, Cathcart R, Schwiers E, Hochstein P. Uric acid provides an antioxidant defense in humans against oxidant- and radical- caused aging and cancer: A hypothesis. Proc Natl Acad Sci U S A. 1981; 78:68586862.

39. Church WH, Ward VL. Uric acid is reduced in the substantia nigra in Parkinson's Diseases: Effect on dopamine oxidation. Brain Res Bull. 1994; 33:419-425.

40. Bogdanov M, Matson WR, Wang L, Matson T, SaundersPullman R, Bressman SS, Beal MF. Metabolomic profiling to develop blood biomarkers for Parkinson's disease. Brain. 2008; 131:389-396.

41. Andreadou E, Nikolaou C, Gournaras F, Rentzos M, Boufidou F, Tsoutsou A, Zournas C, Zissimopoulos V, Vassilopoulos D. Serum uric acid levels in patients with Parkinson's disease: Their relationship to treatment and disease duration. Clin Neurol Neurosurg. 2009; 111:724728.

42. Johansen KK, Wang L, Aasly JO, White LR, Matson WR, Henchcliffe C, Beal MF, Bogdanov M. Metabolomic profiling in LRRK2-related Parkinson's disease. PLoS One. 2009; 4:e7551.

43. Cipriani S, Chen X, Schwarzschild MA. Urate: A novel biomarker of Parkinson's disease risk, diagnosis and prognosis. Biomark Med. 2010; 4:701-712.

44. Shen L, Ji HF. Low uric acid levels in patients with Parkinson's disease: Evidence from meta-analysis. BMJ Open. 2013; 3:e003620.

45. Chahine LM, Stern MB, Chen-Plotkin A. Blood-based biomarkers for Parkinson's disease. Parkinsonism Relat Disord. 2014; 20 (Suppl 1):S99-103.

46. Weisskopf MG, Reilly EO, Chen H, Schwarzschild MA, Ascherio A. Plasma urate and risk of Parkinson's Disease. Am J Epidemiol. 2007; 166:561-567.

47. Fang P, Li X, Luo JJ, Wang H, Yang XF. A double-edged sword: Uric acid and neurological disorders. Brain Disord Ther. 2013; 2:e109.

48. Matsuo T, Ishikawa Y. Protective role of uric acid against photooxidative stress in the silkworm, Bombyx mori (Lepidoptera: Bombycidae). Appl Entomol Zool. 1999; $34: 481-484$.

(Received January 28, 2016; Accepted February 2, 2016) 